\title{
Shot Noise Induced by Nonequilibrium Spin Accumulation
}

\author{
Tomonori Arakawa, ${ }^{1, *}$ Junichi Shiogai, ${ }^{2}$ Mariusz Ciorga,${ }^{3}$ Martin Utz, ${ }^{3}$ Dieter Schuh, ${ }^{3}$ Makoto Kohda, ${ }^{2,4}$ \\ Junsaku Nitta, ${ }^{2}$ Dominique Bougeard, ${ }^{3}$ Dieter Weiss, ${ }^{3}$ Teruo Ono, ${ }^{5}$ and Kensuke Kobayashi ${ }^{1, \dagger}$ \\ ${ }^{1}$ Department of Physics, Graduate School of Science, Osaka University, 1-1 Machikaneyama, Toyonaka, $560-0043$ Osaka, Japan \\ ${ }^{2}$ Department of Materials Science, Tohoku University, 980-8579 Sendai, Miyagi, Japan \\ ${ }^{3}$ Institute of Experimental and Applied Physics, University of Regensburg, D-93040 Regensburg, Germany \\ ${ }^{4}$ PRESTO, Japan Science and Technology Agency, 332-0012 Kawaguchi, Saitama, Japan \\ ${ }^{5}$ Institute for Chemical Research, Kyoto University, 611-0011 Uji, Kyoto, Japan
}

(Received 16 May 2014; published 7 January 2015)

\begin{abstract}
When an electric current passes across a potential barrier, the partition process of electrons at the barrier gives rise to the shot noise, reflecting the discrete nature of the electric charge. Here we report the observation of excess shot noise connected with a spin current which is induced by a nonequilibrium spin accumulation in an all-semiconductor lateral spin-valve device. We find that this excess shot noise is proportional to the spin current. Additionally, we determine quantitatively the spin-injection-induced electron temperature by measuring the current noise. Our experiments show that spin accumulation driven shot noise provides a novel means of investigating nonequilibrium spin transport.
\end{abstract}

DOI: 10.1103/PhysRevLett.114.016601

In 1918, Schottky argued that the electric flow in a vacuum tube fluctuates in a unique way such that the spectral density of the fluctuations is proportional to the unit of charge $e(e>0)$ and to the mean current [1]. This is the shot noise, the direct consequence of the discreteness of the electron charge. Now, as an electron possesses not only charge but also spin, one may ask how the discreteness of electron spin affects the current fluctuations. Although such spin-dependent shot noise has been discussed theoretically in various contexts [2-11], it has never been evidenced experimentally.

Recently it was pointed out that a nonequilibrium spin accumulation, which can be generated, e.g., by electrical spin injection, modifies the current noise spectrum and allows measurement of the magnitude of the spin accumulation electrically [11]. While Meair et al. explore in this theoretical work the noise measured between different contacts of a mesoscopic cavity with and without nonequilibrium spin accumulation, we analyze here noise measured across a tunneling barrier where one of the contacts carries a nonequilibrium spin accumulation while the other is in equilibrium [see inset of Fig. 1(a)]. Assuming a linear $I-V$ characteristic of the device, the current spectral density $S$ is for $\Delta \mu=0$ proportional to the applied voltage $V$ due to shot noise. For finite spin accumulation, however, $S$ is for $T=0$ given by $S \propto|e V+\Delta \mu / 2|+|e V-\Delta \mu / 2|$. This means that at $V=0$ the noise density is finite. Plotted as a function of $V$, the spin accumulation appears as a plateau of width $\Delta \mu / e$, shown in Fig. 1(a). Finite temperatures will smear out the pronounced kinks at $\pm \Delta \mu / 2 e$. Using instead of an unpolarized contact in thermal equilibrium a ferromagnetic one offers even more options to measure the spin accumulation electrically, as is pointed
PACS numbers: 72.25.Dc, 72.15.Lh, 72.25.Hg, 73.40.Ty

out below. In the case of a ferromagnetic contact, the expression for $S$ gets modified and reads

$$
S_{\uparrow / \downarrow} \propto \alpha|e V \pm \Delta \mu / 2|+\beta|e V \mp \Delta \mu / 2| .
$$

Here $\alpha$ and $\beta(\alpha>\beta)$ define the ratio of the tunnel conductances of majority and minority spins $(\alpha+\beta=1)$, and $\uparrow / \downarrow$ denotes the spin direction with respect to the magnetization of the detector contact. The resulting $S(V)$ relation is plotted in Fig. 1(b). In addition to the kinks at $\pm \Delta \mu / 2 e$, the spin-up and spin-down noise density is horizontally shifted by $\gamma \Delta \mu / e$, where $\gamma$ is the spin asymmetry coefficient given by $\gamma=\alpha-\beta$. Below we denote the vertical shift of $S_{\uparrow}$ and $S_{\downarrow}$ as excess shot noise, corresponding to the noise which is entirely generated by the spin accumulation.

In this Letter we demonstrate the validity of the concepts presented above by measuring shot noise across a tunneling
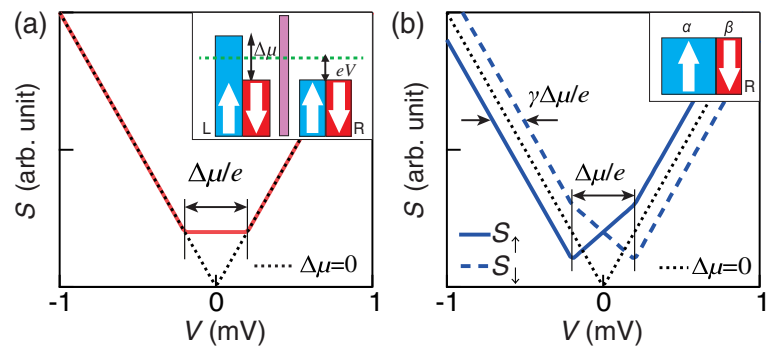

FIG. 1 (color online). (a) Current noise at $T=0$ for $\Delta \mu=400 \mu \mathrm{eV}$, when a nonmagnetic detector electrode is used [11]. The inset shows the schematic energy diagram. The dotted curve indicates shot noise without spin accumulation. (b) Current noise when a ferromagnetic contact is used on one side of the tunneling barrier as a detector electrode ( $\alpha=0.75$ and $\beta=0.25$ ). 
barrier in the presence of spin accumulation. By using a ferromagnetic detector electrode, we have successfully extracted the relation between the spin current and the corresponding excess shot noise, and we have found that the estimated Fano factor directly shows that the spin degree of freedom is preserved in the tunneling process. Given the importance of shot noise in various fields, especially in device technology [12] and mesoscopic physics [13-15], excess shot noise due to spin accumulation could not only serve as a unique probe to explore the spin-dependent nonequilibrium transport process but also to shed new light on the recently emerging field of spin noise spectroscopy [16-19].

A spin accumulation can be generated, e.g., by electrical spin injection [20,21]. To this end, we prepared a lateral all-semiconductor spin-valve device, which was fabricated from a single epitaxial wafer grown by molecular beam epitaxy on a (001) GaAs substrate, consisting of, in the growth order, a GaAs buffer, an AlGaAs/GaAs superlattice, a $1 \mu \mathrm{m} \mathrm{n}$-GaAs channel, a $15 \mathrm{~nm}$ GaAs with linearly graded doping $n \rightarrow n^{+}\left(n=2 \times 10^{16} \mathrm{~cm}^{-3}\right.$ and $n^{+}=$ $\left.5 \times 10^{18} \mathrm{~cm}^{-3}\right)$, an $8 \mathrm{~nm} n^{+}$-GaAs, a $2.2 \mathrm{~nm} \mathrm{AlGaAs}$, and a $50 \mathrm{~nm}\left(\mathrm{Ga}_{0.945} \mathrm{Mn}_{0.055}\right)$ As. Because of the high $n^{+}$doped GaAs region adjacent to the degenerately $p$-doped (Ga,Mn)As layer, a tunneling Esaki diode structure is formed at the junction, enabling efficient generation and detection of spin accumulation in $n$-GaAs. The wafer was patterned into $50 \mu \mathrm{m}$-wide mesas along the [110] direction by standard photolithography and wet chemical etching. Then six ferromagnetic $(\mathrm{Ga}, \mathrm{Mn}) \mathrm{As}$ electrodes ( $E 1$ to $E 6)$ are defined by electron beam lithography and by wet chemical etching down to the lightly doped $n$-GaAs channel, as schematically shown in Fig. 2(a) [22-24]. Each ferromagnetic electrode serves a specific purpose: one is used as an injection electrode (E2), three as detection electrodes (E3,E4, and $E 5)$, and two as reference electrodes ( $E 1$ and $E 6)$. Only the $E 1$ electrode is grounded.

By applying a constant current $I_{\text {inj }}$ to $E 2$ [see Fig. 2(a)], we inject spin polarized electrons into the $n$-GaAs channel. As a consequence, spin accumulation-i.e., splitting of the chemical potentials $\left(\Delta \mu=\mu_{\uparrow}-\mu_{\downarrow}\right)$ for spin-up and spin-down electrons ( $\mu_{\uparrow}$ and $\mu_{\downarrow}$, respectively)—occurs underneath the injection contact and diffuses to both sides. Figure 2(b) shows a typical spin accumulation signal obtained by measuring the nonlocal dc voltage difference between contacts $E 3$ and $E 6$ as a function of the in-plane magnetic field $B$ [see Fig. 2(a)]. All of the measurements were carried out at $1.6 \mathrm{~K}$ in a variable temperature insert. The abrupt voltage changes, displayed in Fig. 1(b), correspond to magnetization switching of E2 or E3. Different coercive fields were adjusted by different widths of the respective contacts. The voltage change $\Delta V$ between parallel (P) and antiparallel (AP) magnetization configurations, being $0.30 \mathrm{mV}$ here, is proportional to $\Delta \mu$ and
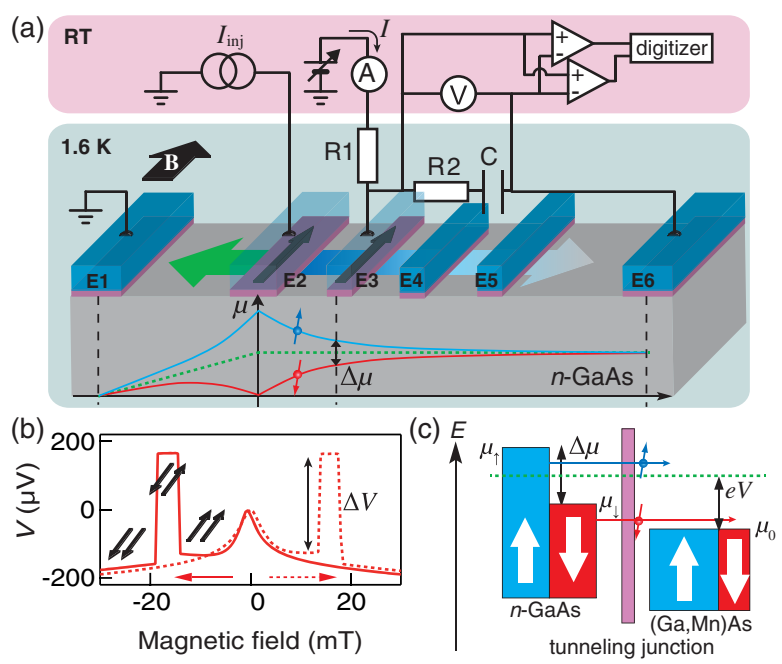

FIG. 2 (color online). (a) Schematic diagram of the sample and measurement system. Six (Ga,Mn)As electrodes ( $E 1$ to $E 6)$ are placed on the $n$-GaAs channel, where $E 2(4 \mu \mathrm{m} \times 50 \mu \mathrm{m}$ size $)$ is an injection electrode, while either $E 3, E 4$, or $E 5(0.5 \mu \mathrm{m} \times$ $50 \mu \mathrm{m}$ size) is a detection electrode. The center-to-center spacing between the neighboring electrodes is $5 \mu \mathrm{m}$. Schematic spatial dependence of each chemical potential in the $n$-GaAs channel is illustrated. (b) Typical nonlocal voltage signal for $I_{\text {inj }}=-23 \mu \mathrm{A}$. A peak observed around the zero magnetic field is induced by dynamic nuclear spin polarization (DNP) [23]. This effect is irrelevant to the present result, as the noise measurement was performed outside of the DNP region. (c) Schematic energy diagram at the detection electrode in the presence of $e V$ and $\Delta \mu$.

given by $\Delta V=\gamma \Delta \mu / e[25,26]$. For our device we extract $\gamma=0.82 \pm 0.03$ in the small $I_{\text {inj }}$ limit $[22,24]$.

To measure $S$ through the tunneling barrier formed by the Esaki diode at the $p$-(Ga,Mn)As $/ n$-GaAs interface at the corresponding contact, we use an additional circuit, as shown in Fig. 2(a). Three passive components are placed at $1.6 \mathrm{~K}$ : two surface mounted metal-film resistors $(R 1=1 \mathrm{M} \Omega, R 2=1 \mathrm{k} \Omega)$ and a laminated ceramic capacitor $(C=1 \mu \mathrm{F}) . S$ is converted to voltage noise by $R 2$, while $R 1$ prevents the current noise from leaking to the voltage source. The two sets of voltage noise signals are independently amplified by two amplifiers (NF LI-75A) at room temperature and are recorded at a two-channel digitizer. Cross-correlation spectra were obtained in the frequency range between $16 \mathrm{kHz}$ and $160 \mathrm{kHz}(9001$ points) $[27,28]$. To extract $S$ across the tunneling barrier, we carefully calibrated the measurement system and eliminated thermal noise from $R 2$ and the channel resistance [24]. We experimentally confirmed that the contribution of frequency-dependent noise, such as $1 / f$ noise, does not affect $S$ for the parameter range examined.

The measurement procedure is as follows [24]: together with the constant spin injection current $I_{\text {inj }}$, which determines the manitude of the spin accumulation, we inject a small current $I$ to one of the detection electrodes 
(either E3, E4, or E5) using a constant current technique [see Fig. 2(a)]. We directly measure the voltage drop $V$ and $S$ across the tunneling barrier, as schematically shown in Fig. 2(c). The dependence of these values on current $I$ was measured for both $\mathrm{P}$ and AP configurations. Note that by reversing the contact magnetization we switch the up and down direction of the accumulated spins in the $n$-GaAs channel. As $I$ is set between $-300 \mathrm{nA}$ and $300 \mathrm{nA}$, well below $I_{\text {inj }}$, the influence of this probe current on the spin accumulation is negligibly small. The data shown in Fig. 3(a) are obtained for injection currents $I_{\text {inj }}=0,-9$, and $-23 \mu \mathrm{A}$ at $E 3$. In order to compare our data with the predictions of Eq. (1), we discuss below the measured noise $S$ as a function of $V$. For $I_{\text {inj }}=0 \mu \mathrm{A}$, the observed $S$ is independent of the magnetization configuration, and it monotonically increases as $|V|$ increases, as expected from conventional shot noise theory [13]. The finite $S$ at $V=0$ stems from thermal noise. This curve is perfectly reproduced by the conventional formula given by

$$
S=\frac{4 k_{\mathrm{B}} T_{e}}{R_{\mathrm{d}}}+2 F\left(e I \operatorname{coth}\left(\frac{e V}{2 k_{\mathrm{B}} T_{e}}\right)-\frac{4 k_{\mathrm{B}} T_{e}}{R_{\mathrm{d}}}\right)
$$

where $R_{\mathrm{d}}, T_{e}$, and $F$ are the differential resistance at a given bias, the electron temperature, and the Fano factor, respectively. For $T_{e}=1.6 \mathrm{~K}$, the fitting to this formula gives $F=0.78 \pm 0.04$, as shown in Fig. 3(a) by the dotted curve. The Fano factor is slightly reduced from unity, as expected for a tunneling junction. This is most probably due to the contribution of defects in the tunneling barrier which are unavoidably created during the (low-temperature) growth process [29-31].

When $I_{\text {inj }}$ is finite, i.e., for -9 and $-23 \mu \mathrm{A}, S$ starts to depend on the magnetization configuration, as expected by Eq. (1) except for the low bias region, which is dominated by thermal noise [See Figs. 1(b) and 3(a)]. Moreover, for $I_{\text {inj }}=-23 \mu \mathrm{A}$, the horizontal shift of $S$ between $\mathrm{P}$ and AP configurations is $0.28 \mathrm{mV}$, matching closely the magnitude of the nonlocal voltage signal of $\Delta V=0.30 \mathrm{mV}$, shown in Fig. 1(b). This indicates the validity of the scenario put forward in the introduction and demonstrates that "noise spectroscopy" successfully detects the nonequilibrium spin accumulation $\Delta \mu$ [see also Fig. 2(c)]. We emphasize that the observed difference between the two different magnetic configurations is reproducible. Actually, the $B$ dependence of $S$ was measured for a constant $V=-6.8 \mathrm{mV}$ in independent measurements, as shown in Fig. 3(b). Clear spin-valve-like changes were observed for $I_{\text {inj }}=-9 \mu \mathrm{A}$ and $-23 \mu \mathrm{A}$, reflecting magnetization switching in the electrodes, while no detectable change was observed for $I_{\text {inj }}=0 \mu \mathrm{A}$. Thus, the configuration-dependent contribution of the noise measured at finite $I_{\text {inj }}$ is undoubtedly the shot noise associated with spin accumulation.
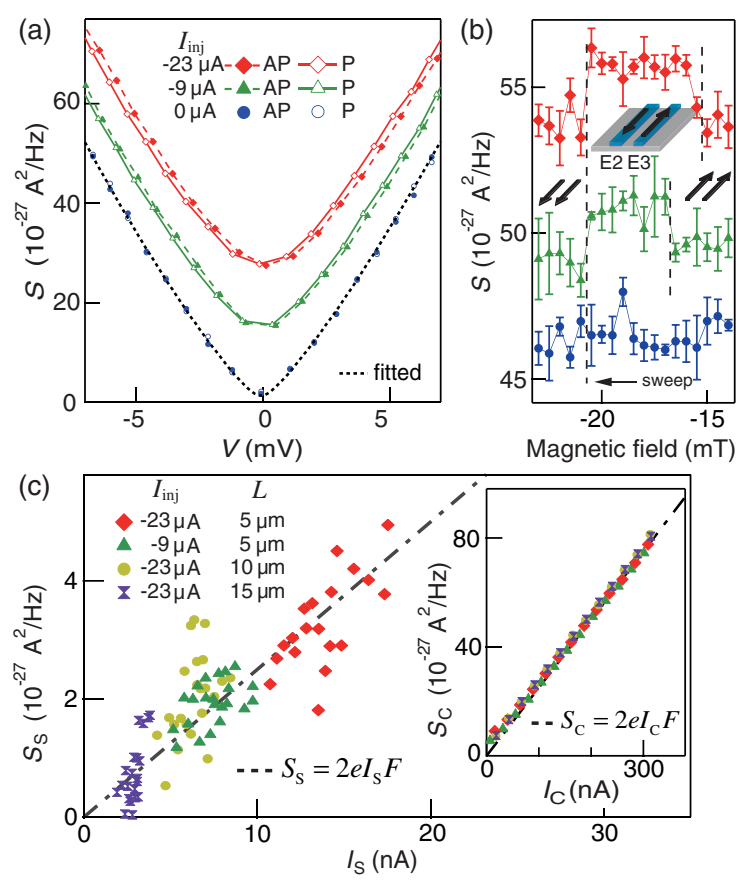

FIG. 3 (color online). (a) Measured $S$ at $E 3$ as a function of $V$ for $\mathrm{P}$ and AP configurations for several injection currents. The curves are offset vertically by $1 \times 10^{-26} \mathrm{~A}^{2} / \mathrm{Hz}$ for clarity. The dotted curve is the fitted curve from Eq. (1). The error bar for each point is $\pm 0.4 \times 10^{-27} \mathrm{~A}^{2} / \mathrm{Hz}$. (b) $B$ dependence of $S$ measured with keeping $V$ constant $(V=-6.8 \mathrm{mV})$ for $I_{\text {inj }}=0,-9$, and $-23 \mu \mathrm{A}$ (from bottom to top). The thick arrows denote the magnetization directions of $E 2$ and $E 3$. (c) $S_{S}$ versus $I_{S}$ for the bias region $\left(|e V|>\Delta \mu / 2,2 k_{\mathrm{B}} T\right)$ for several injection currents and for different detection electrodes. The dashed line is the linear relation with $F=0.77$. The inset shows the counterpart of the main graph for $S_{C}$ versus $I_{C}$.

In order to distinguish contributions from the voltage drop across the barrier $V$ and the spin accumulation in the channel $\Delta \mu$ in the measured current, we adopt the following model based on the Landauer-Büttiker formalism [13,32]. Currents for $\mathrm{P}\left(I_{\mathrm{P}}\right)$ and $\mathrm{AP}\left(I_{\mathrm{AP}}\right)$ states are written as

$$
I_{\mathrm{P} / \mathrm{AP}}=\frac{e}{h} T_{1}(e V \pm \Delta \mu / 2)+\frac{e}{h} T_{2}(e V \mp \Delta \mu / 2),
$$

with $T_{1(2)}$ being the sum of all of the transmission probabilities of the tunneling channels from the nonmagnetic GaAs conduction band states to the majority (minority) spin states in the ferromagnetic $(\mathrm{Ga}, \mathrm{Mn}) \mathrm{As}$. The first and second terms in Eq. (3) represent tunneling currents with up- and down-spin, respectively. By using the ratio of the tunneling conductances, $\alpha=T_{1} /\left(T_{1}+T_{2}\right)$ and $\beta=T_{2} /\left(T_{1}+T_{2}\right)$, Eq. (3) can be rewritten as $I_{\mathrm{P} / \mathrm{AP}}=$ $(2 e / h) \bar{T} e V \pm(e / h)(\alpha-\beta) \bar{T} \Delta \mu$, where $\bar{T} \equiv\left(T_{1}+T_{2}\right) / 2$. To separate the currents driven by the applied bias $V$ and the spin accumulation $\Delta \mu$, we define the charge current $I_{C} \equiv(2 e / h) \bar{T} e V$ and the spin current $I_{S} \equiv(e / h) \bar{T} \Delta \mu$. Note that this spin current is solely driven by the spin 
accumulation in the $n$-GaAs channel. These currents can be extracted from the experiment using the relations, $\left\langle I_{C}\right\rangle=\left(I_{\mathrm{P}}+I_{\mathrm{AP}}\right) / 2$ and $\left\langle I_{S}\right\rangle=\left(I_{\mathrm{P}}-I_{\mathrm{AP}}\right) / 2(\alpha-\beta)$. By using the experimentally obtained value of $\gamma$, we determined $\alpha$ and $\beta$ as 0.91 and 0.09 , respectively. The energy dependence of these values is negligible in the present experiment at low bias.

We first focus on the excess shot noise in the high bias region $\left(|e V|>\Delta \mu / 2,2 k_{B} T\right)$ and discuss the measured noise in parallel configuration $S_{\mathrm{P}}$ and antiparallel configuration $S_{\mathrm{AP}}$. Note that, because $|e V|>\Delta \mu / 2$, either $e V \pm \Delta \mu / 2>$ 0 or $e V \pm \Delta \mu / 2<0$ holds [see Fig. 2(c)]. By similar transformations, $S_{\mathrm{P}}$ and $S_{\mathrm{AP}}$ can be written as $S_{\mathrm{P} / \mathrm{AP}}=$ $\left(4 e^{2} / h\right) \bar{T} F e V \pm\left(2 e^{2} / h\right)(\alpha-\beta) \bar{T} F \Delta \mu$. Accordingly, the conventional shot noise $S_{C}$ and the excess shot noise $S_{S}$ are defined as $S_{C} \equiv\left(4 e^{2} / h\right) \bar{T} F e V=\left(S_{\mathrm{P}}+\mathrm{S}_{\mathrm{AP}}\right) / 2$ and $S_{S} \equiv\left(2 e^{2} / h\right) \bar{T} F \Delta \mu=\left(\left|S_{\mathrm{P}}-S_{\mathrm{AP}}\right| / 2(\alpha-\beta)\right)$, respectively.

Using experiments such as the one shown in Fig. 2(a), we can experimentally derive the relation between $\left\langle I_{S}\right\rangle$ and $S_{S}$ exactly as in conventional shot noise experiments. By tuning $I_{\mathrm{inj}}$, choosing different electrodes for detection (E3, $E 4$, or E5), or both, we perform noise measurements for different values of $\Delta \mu$. In Fig. 3(c), we show the corresponding data $S_{S}$ vs $\left\langle I_{S}\right\rangle$ and $S_{C}$ vs $\left\langle I_{C}\right\rangle$ for $|e V|>\Delta \mu / 2$, $2 k_{B} T$, for several values of $I_{\text {inj }}$ and for different detection electrodes. $S_{C}$ is well fitted by the linear function $S_{C}=2 e F\left|\left\langle I_{C}\right\rangle\right|$ [see inset in Fig. 3(c)], which yields $F=0.77 \pm 0.04$, consistent with the zero-injection case discussed above [see Fig. 3(a)]. Similarly, we find that the excess shot noise $S_{S}$ also linearly depends on $\left\langle I_{S}\right\rangle$ and, more importantly, satisfies the relation $S_{S}=2 e F\left|\left\langle I_{S}\right\rangle\right|$ (dashed line) with the same Fano factor obtained for the conventional shot noise. Both the linear relations and the equal Fano factors justify the applied procedure to extract the excess shot noise which is generated by the spin accumulation.

In the low bias region $(|e V| \leq \Delta \mu / 2), S_{\mathrm{P}}$ and $S_{\mathrm{AP}}$ are expected to have opposite slopes, as shown in Fig. 1(b). For finite temperature, the pronounced kinks at $V= \pm \Delta \mu / 2 e$ are smeared, and Eq. (1) need to be replaced by $S_{\uparrow / \downarrow} \propto$ $\alpha|e V \pm \Delta \mu / 2| \cosh \left((e V \pm \Delta \mu / 2) / 2 k_{\mathrm{B}} T_{e}\right)+\beta|e V \mp \Delta \mu / 2|$ $\cosh \left((e V \mp \Delta \mu / 2) / 2 k_{\mathrm{B}} T_{e}\right)$. For an electron temperature of $T_{e}=1.6 \mathrm{~K}$, equal to the bath temperature, the kinks should be detectable [11]. However, this structure is not resolved in experiments due to the thermal smearing of the distribution function by the injected hot electrons.

The latter can be seen by estimating the degree of nonequilibrium in terms of the effective electron temperature by fitting $\left(S_{\mathrm{P}}+S_{\mathrm{AP}}\right) / 2$ to Eq. (2). The estimated value of $k_{\mathrm{B}} T_{e}$ is always larger than $\Delta \mu . k_{\mathrm{B}} \Delta T_{e}$ and $\Delta \mu$ are plotted against $I_{\text {inj }}$ and the injector-detector separation $L$ in Figs. 4(a) and 4(b), respectively, where $\Delta T_{e}\left(\equiv T_{e}-1.6 \mathrm{~K}\right)$ is the effective temperature rise. One can see that, as $\Delta \mu$ increases as a function of $I_{\mathrm{inj}}, k_{\mathrm{B}} \Delta T_{e}$ increases similarly. To the best of our knowledge, direct information such as
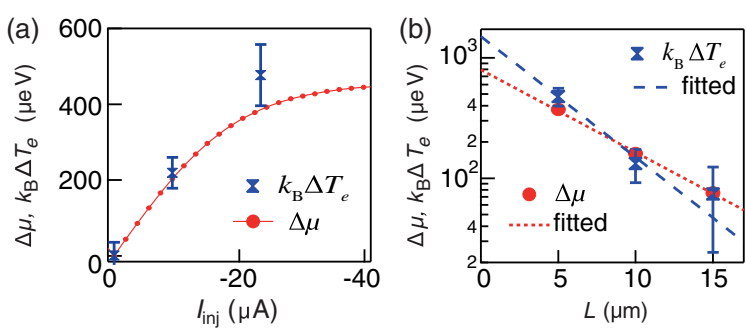

FIG. 4 (color online). (a) $I_{\text {inj }}$ dependence of the extracted $k_{\mathrm{B}} \Delta T_{e}$ and $\Delta \mu$ at detector $E 3$. (b) $L$ dependence of $k_{\mathrm{B}} \Delta T_{e}$ and $\Delta \mu$ for $I_{\text {inj }}=-23 \mu \mathrm{A}$.

$k_{\mathrm{B}} \Delta T_{e}$ to characterize the degree of nonequilibrium due to spin accumulation has not been demonstrated so far. Moreover, as $\Delta \mu$ relaxes according to the diffusion equation, $k_{\mathrm{B}} \Delta T_{e}$ also relaxes. By fitting $\Delta \mu$ and $k_{\mathrm{B}} \Delta T_{e}$ to $\Delta \mu \propto$ $\exp \left(-L / \lambda_{S}\right)$ and $k_{\mathrm{B}} \Delta T_{e} \propto \exp \left(-L / \lambda_{e}\right)$, we obtain a spin relaxation length $\lambda_{S}=5.6 \mu \mathrm{m}$ and an energy relaxation length $\lambda_{e}=4.3 \mu \mathrm{m}$ in the channel, respectively [see the dashed curves in Fig. 4(b)]. The good agreement between the two relaxation lengths indicates that spin relaxation is accompanied by energy relaxation.

Finally, we note that the $S_{C}$ and $S_{S}$ described here are identical due to the absence of spin flips $[2,4,8,10]$ or Coulomb interaction effects [6], which would otherwise generate different Fano factors for spin and charge current. By measuring this difference, general spin-dependent shot noise physics can be addressed. In addition, the present demonstration can lead to a new probe to explore the spintransfer torque physics [9], the spin heat accumulation [33], and the spin-dependent chiral edge states in topological insulators [34].

In summary, we have shown that a nonequilibrium spin accumulation can be detected in the noise spectrum including the distribution function. The Landauer-Büttiker formalism allowed us to disentangle conventional shot noise associated with the charge current and excess shot noise, connected with a spin current. However, because of the elevated electron temperature stemming from nonlocal spin injection, it was not possible to explore excess shot noise in the limit $V \rightarrow 0$, i.e., when only a pure spin current is flowing.

We appreciate the fruitful comments and support from M. Ferrier and R. Sakano. This work is partially supported by a Grant-in-Aid for Scientific Research (S) (Grant No. 26220711) from JSPS, a Grant-in-Aid for Scientific Research on Innovative Areas "Fluctuation and Structure" (Grant No. 25103003) from MEXT, and a Grant-in-Aid for Young Scientists (Start-up) (Grant No. 25887037) from JSPS, the Murata Science Foundation. D. W. and J. N. acknowledge support from the German and Japanese Joint Research Program, the German Science Foundation (DFG) via SFB 689, and the Collaborative Research Program of the Institute for Chemical Research, Kyoto University. 
*arakawa@phys.sci.osaka-u.ac.jp

${ }^{\dagger}$ kensuke@phys.sci.osaka-u.ac.jp

[1] W. Schottky, Ann. Phys. (Berlin) 362, 541 (1918).

[2] E. G. Mishchenko, Phys. Rev. B 68, 100409 (2003).

[3] W. Belzig and M. Zareyan, Phys. Rev. B 69, 140407(R) (2004).

[4] A. Lamacraft, Phys. Rev. B 69, 081301(R) (2004).

[5] B. Wang, J. Wang, and H. Guo, Phys. Rev. B 69, 153301 (2004).

[6] O. Sauret and D. Feinberg, Phys. Rev. Lett. 92, 106601 (2004).

[7] J. Foros, A. Brataas, Y. Tserkovnyak, and G. E. W. Bauer, Phys. Rev. Lett. 95, 016601 (2005).

[8] R. L. Dragomirova and B. K. Nikolić, Phys. Rev. B 75, 085328 (2007).

[9] A. L. Chudnovskiy, J. Swiebodzinski, and A. Kamenev, Phys. Rev. Lett. 101, 066601 (2008).

[10] R. L. Dragomirova, L. P. Zârbo, and B. K. Nikolić, Europhys. Lett. 84, 37004 (2008).

[11] J. Meair, P. Stano, and P. Jacquod, Phys. Rev. B 84, 073302 (2011).

[12] S. M. Sze and K. K. Ng, Physics of Semiconductor Devices (John Wiley \& Sons, New York, 2007).

[13] Ya. M. Blanter and M. Büttiker, Phys. Rep. 336, 1 (2000).

[14] Quantum Noise in Mesoscopic Physics, edited by Y. V. Nazarov (Kluwer, Dordrecht, 2003).

[15] C. W. J. Beenakker and C. Schönenberger, Phys. Today 56, No. 5, 37 (2003).

[16] S. A. Crooker, D. G. Rickel, A. V. Balatsky, and D. L. Smith, Nature (London) 431, 49 (2004).

[17] M. Oestreich, M. Romer, R. J. Haug, and D. Hagele, Phys. Rev. Lett. 95, 216603 (2005).

[18] S. A. Crooker, J. Brandt, C. Sandfort, A. Greilich, D. R. Yakovlev, D. Reuter, A. D. Wieck, and M. Bayer, Phys. Rev. Lett. 104, 036601 (2010).
[19] A. V. Kuhlmann, J. Houel, A. Ludwig, L. Greuter, D. Reuter, A. D. Wieck, M. Poggio, and R. J. Warburton, Nat. Phys. 9, 570 (2013).

[20] F. J. Jedema, A. T. Filip, and B. J. van Wees, Nature (London) 410, 345 (2001).

[21] X. Lou, C. Adelmann, S. A. Crooker, E. S. Garlid, J. Zhang, K. S. Madhukar Reddy, S. D. Flexner, C. J. Palmstrøm, and P. A. Crowell, Nat. Phys. 3, 197 (2007).

[22] M. Ciorga, A. Einwanger, U. Wurstbauer, D. Schuh, W. Wegscheider, and D. Weiss, Phys. Rev. B 79, 165321 (2009).

[23] J. Shiogai, M. Ciorga, M. Utz, D. Schuh, T. Arakawa, M. Kohda, K. Kobayashi, T. Ono, W. Wegscheider, D. Weiss, and J. Nitta, Appl. Phys. Lett. 101, 212402 (2012).

[24] See Supplemental Material at http://link.aps.org/ supplemental/10.1103/PhysRevLett.114.016601 for additional data and discussion.

[25] A. Fert and H. Jaffrès, Phys. Rev. B 64, 184420 (2001).

[26] S. Takahashi and S. Maekawa, Phys. Rev. B 67, 052409 (2003).

[27] A. Kumar, L. Saminadayar, D. C. Glattli, Y. Jin, and B. Etienne, Phys. Rev. Lett. 76, 2778 (1996).

[28] T. Arakawa, K. Sekiguchi, S. Nakamura, K. Chida, Y. Nishihara, D. Chiba, K. Kobayashi, A. Fukushima, S. Yuasa, and T. Ono, Appl. Phys. Lett. 98, 202103 (2011).

[29] R. M. Feenstra, J. M. Woodall, and G. D. Pettit, Phys. Rev. Lett. 71, 1176 (1993).

[30] T. Tsuruoka, N. Tachikawa, S. Ushioda, F. Matsukura, K. Takamura, and H. Ohno, Appl. Phys. Lett. 81, 2800 (2002).

[31] A. Richardella, P. Roushan, S. Mack, B. Zhou, D. A. Huse, D. D. Awschalom, and A. Yazdani, Science 327, 665 (2010).

[32] S. Datta, Electronic Transport in Mesoscopic Systems (Cambridge University Press, Cambridge, England, 1997).

[33] F. K. Dejene, J. Flipse, G. E. W. Bauer, and B. J. van Wees, Nat. Phys. 9, 636 (2013).

[34] M. Z. Hasan and C. L. Kane, Rev. Mod. Phys. 82, 3045 (2010). 